\title{
Influence of Processing Method on the Mechanical and Electrical Properties of MWCNT/PET Composites
}

\author{
O. Rodríguez-Uicab, ${ }^{1}$ A. May-Pat, ${ }^{1}$ F. Avilés, ${ }^{1}$ P. Toro, ${ }^{2}$ \\ and $M$. Yazdani-Pedram ${ }^{3}$ \\ ${ }^{1}$ Unidad de Materiales, Centro de Investigación Científica de Yucatán, A.C., Calle 43 No. 130, Colonia Chuburná de Hidalgo, \\ 97200 Mérida, Yucatán, Mexico \\ ${ }^{2}$ Facultad de Ciencias Físicas y Matemáticas, Universidad de Chile, Beaucheff 861, Santiago, Chile \\ ${ }^{3}$ Facultad de Ciencias Químicas y Farmacéuticas, Universidad de Chile, Olivos 1007, Santiago, Chile
}

Correspondence should be addressed to O. Rodríguez-Uicab; omarrodriguezuicab@hotmail.com

Received 15 November 2012; Accepted 12 December 2012

Academic Editor: Iwan Kityk

Copyright (C) 2013 O. Rodríguez-Uicab et al. This is an open access article distributed under the Creative Commons Attribution License, which permits unrestricted use, distribution, and reproduction in any medium, provided the original work is properly cited.

Multiwalled carbon nanotube (MWCNT)/polyethylene terephthalate (PET) composites were prepared by three processing methods: direct extrusion (DE), melt compounding followed by extrusion (MCE), and dispersion of the MWCNTs in a solvent by sonication followed by extrusion (SSE). The mechanical properties of the MWCNT/PET composites processed by MCE increased with $0.1 \mathrm{wt} \%$ MWCNTs with respect to the neat PET. The electrical percolation threshold of MWCNT/PET composites processed by $\mathrm{DE}$ and MCE was $~ 1 \mathrm{wt} \%$ and the conductivity was higher for composites processed by MCE. Raman spectroscopy and scanning electron microscopy showed that mixing the MWCNTs by melt compounding before extruding yields better dispersion of the MWCNTs within the PET matrix. The processing method assisted by a solvent resulted in matrix plasticization.

\section{Introduction}

Polyethylene terephthalate (PET) is a thermoplastic of major industrial importance due to its high performance, low cost, and good physical properties. It has a variety of applications such as textiles, fibers, films, bottle containers, and food packaging and is also used in the automobile and electronic industries $[1,2]$. On the other hand, the discovery of carbon nanotubes (CNTs) has attracted much attention in different areas of science and technology, from fundamental science to materials engineering. The excellent electrical, thermal, and mechanical properties of CNTs, together with their high aspect ratios and large surface areas, make them ideal candidates as reinforcements for thermoplastic polymer composites [3-6]. The most challenging tasks in the fabrication of CNT/polymer composites are a homogeneous dispersion of CNTs in the polymeric matrices and achieving good interfacial interactions between the CNTs and the matrix [7]. Currently, three processing techniques are in common use for fabrication of CNT/polymer composites: in situ polymerization, solution mixing, and melt compounding. Among these three processing techniques, melt compounding has been accepted as a simple and efficient method for processing thermoplastics, especially from an industrial perspective. This method allows the fabrication of high-performance thermoplastic nanocomposites at low cost, facilitating large scale-up for commercial applications. Furthermore, the combination of an inexpensive nonconductive thermoplastic polymer with a very small amount of electrically conductive CNTs is of great technological interest, since this may 
provide attractive possibilities for improving the mechanical and electrical properties of polymer nanocomposites at low manufacturing costs $[5,8,9]$. A common laboratory method for preparing $\mathrm{CNT} /$ thermoplastic composites consists in solution mixing by using a certain solvent which dissolves the polymer and postevaporation of the solvent to form a composite film. The general protocol for all solutioninvolved processing methods includes the dispersion of CNT powder in a liquid medium by vigorous stirring and/or sonication, mixing the CNT dispersion with a dissolved polymer and controlling the evaporation of the solvent with or without vacuum conditions. In general, the most efficient dispersion of CNTs during the first step is achieved by bath or tip sonication [10-12]. Recently, considerable efforts have been devoted to the preparation and study of the influence of CNTs on the mechanical and electrical properties of CNT/thermoplastic composites with melt processable polymer matrices such as polymethyl methacrylate (PMMA) [13], polypropylene (PP) [14], polyethylene (PE) [15], polystyrene (PS) [4], and polyethylene terephthalate (PET) $[5,7-9,16,17]$. Zhu et al. [7] showed that the tensile strength and elastic modulus of MWCNT/PET composites can be improved by using in situ polycondensation. Kim et al. [8] found that MWCNT/PET composites manufactured by melt compounding in a twin-screw extruder improved the mechanical properties of MWCNT/PET composites, and this was attributed to the uniform dispersion of MWCNTs in the PET matrix. They also found that the MWCNTs effectively act as nucleating agents, enhancing the crystallization of PET through heterogeneous nucleation. Santoro et al. [16] dispersed MWCNTs in PET by using a solvent-free method for dispersion followed by melt blending, obtaining MWCNT/PET composites with improved mechanical properties and increased crystallization temperature. Logakis et al. [3] prepared MWCNT/PET composites using three different procedures: in situ polymerization of PET in the presence of MWCNTs, direct melt mixing of PET with MWCNTs, and dilution of an in situ obtained CNT-PET masterbatch in PET by using melt mixing. Using melt mixing they found a very low electrical percolation threshold (0.05-0.10 wt\%) for all composites. Given this background, it is clear that the polymer processing method affects the resulting mechanical and electrical properties of the composites and that the most common methods used for processing these composites are based on melt compounding, extrusion, and/or dissolution. Therefore, the objective of this work is to examine the influence of the polymer processing method on the mechanical and electrical properties of MWCNT/PET composites. To this aim, three processing methods commonly used in the industry and academia were utilized: direct extrusion (DE), melt compounding followed by extrusion (MCE), and MWCNT sonication in solution followed by extrusion (SSE). The composites were characterized by Raman spectroscopy and differential scanning calorimetry (DSC) to investigate the CNT dispersion state and thermal properties, respectively. The results are correlated with the composites fracture surface analyzed by scanning electron microscopy (SEM).

\section{Materials and Methods}

2.1. Materials. The CNTs used were commercial MWCNTs (Baytubes C150P) acquired from Bayer MaterialScience (Leverkusen, Germany) with a purity $>95 \%$, length in the range of $1-4 \mu \mathrm{m}$, outer mean diameter of $13-16 \mathrm{~nm}$, and inner diameter of $4 \mathrm{~nm}$ [18]. These MWCNTs are produced in a high-yield catalytic process based on the chemical vapor deposition method. Phenol (99\% purity) and trichloroethylene (99.5\% purity) were purchased from Sigma Aldrich Corporation (Milwaukee, USA). PET (CLEARTUF 8006) in pellets form was obtained from M\&G Polymers (Houston, USA). CLEARTUF 8006 is a high molecular weight polyethylene terephthalate thermoplastic polymer with intrinsic viscosity of $0.80 \mathrm{dL} / \mathrm{g}$ and a melting point of $250^{\circ} \mathrm{C}$.

2.2. Preparation of MWCNT/PET Composites. PET pellets were first powdered and dried at $150^{\circ} \mathrm{C}$ for $4 \mathrm{~h}$ under vacuum. MWCNT/PET composites were prepared by using three types of processing methods: (i) direct extrusion (DE), (ii) melt compounding in a mixing chamber followed by extrusion (MCE), and (iii) dispersion of the CNTs in a solvent by sonication followed by extrusion (SSE). The procedures used to prepare the composites are schematically shown in Figure 1. All three processing methods have in common the use of extrusion at the last step, which was conducted in a Brabender Plasticorder PL330 twin-screw extruder. The temperature of the heating zone was set to $240^{\circ} \mathrm{C}$ and the screw speed at $30 \mathrm{rpm}$ for all processing methods. Upon completion of the twin-screw extrusion, the extruded circular rods of $\sim 2 \mathrm{~mm}$ diameter were rapidly cooled in a water bath.

For the preparation of MWCNT/PET composites by the DE method, MWCNTs and PET powder were manually mixed and then the mixture was extruded in a Brabender Plasticorder with a conical twin-screw extruder with three heating zones and using a capillary die of $5 \mathrm{~mm}$ internal diameter. The MWCNT/PET composites were obtained as circular rods which were rapidly cooled in a water bath at room temperature placed immediately after the extruder die, see Figure 1(a). Once the composite rods were obtained they were ground in a Brabender mill with a mesh size of $0.78 \mathrm{~mm}$, and then the extrusion process was repeated a second time. Thus, all the materials obtained by the DE method (including neat PET) were processed twice in the extruder.

The MWCNT/PET composites manufactured by MCE, Figure 1(b), employed an additional processing step before extrusion. For this method, the MWCNTs and PET powders were first melt-compounded in a $50 \mathrm{~cm}^{3}$ Banbury Plasticorder mixing chamber at $250^{\circ} \mathrm{C}$. The melt compound process inside the mixing chamber was performed using three incremental mixing speeds. First, the material was placed inside the mixing chamber and mixed for $5 \mathrm{~min}$ at $10 \mathrm{rpm}$, followed by $5 \mathrm{~min}$ at $20 \mathrm{rpm}$, and $60 \mathrm{rpm}$ for $5 \mathrm{~min}$ more. The composites obtained from this melt compounding process were pulverized and dried at $150^{\circ} \mathrm{C}$ for $4 \mathrm{~h}$ and used for direct extrusion following similar conditions as that of 


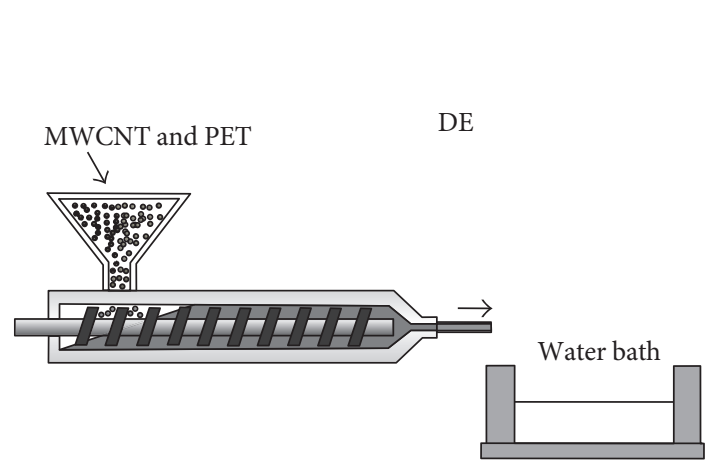

(a)

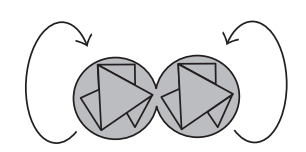

(i) MWCNT/PET pellets

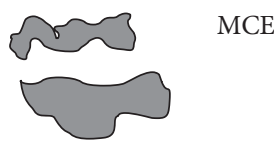

(ii)

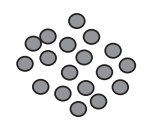

(iii)

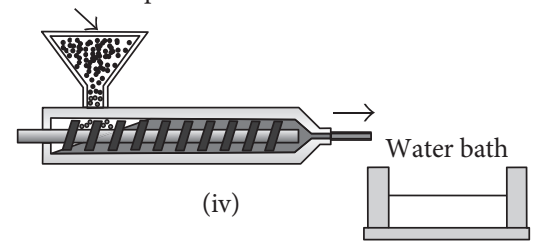

(b)

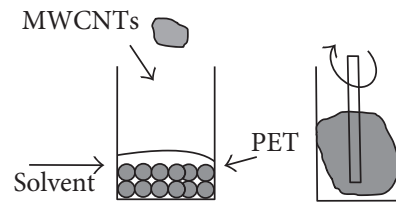

(i)

(ii)

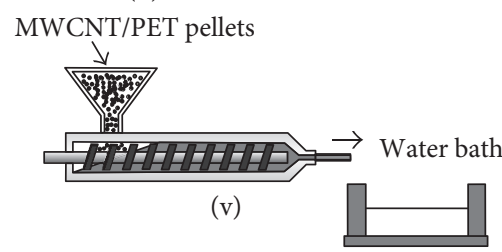

(c)

FIGURE 1: Methods for fabrication of MWCNT/PET composites: (a) DE, (b) MCE, and (c) SSE.

the DE method, with the only difference that for MCE the material passed only once through the extruder.

The MWCNT/PET composites processed by the SSE method, Figure 1(c), also employed an additional step prior to extrusion. In this method, a mixture of phenol and trichloroethylene (60/40 weight ratio) was first prepared at $90^{\circ} \mathrm{C}$ under mechanical agitation for $1 \mathrm{~h}$. The desired amount of MWCNTs was then added to $\sim 20 \mathrm{~mL}$ of the suspension and sonicated for $15 \mathrm{~min}$ using an ultrasonic probe sonicator (VC 750 Sonics and Materials Inc.) of $13 \mathrm{~mm}$ tip diameter and operating at $150 \mathrm{~W}$. The suspension of MWCNTs in phenol/trichloroethylene solution was further stirred on a hot plate at $90^{\circ} \mathrm{C}$ for $30 \mathrm{~min}$. Subsequently, PET powder was added to the $\mathrm{CNT} /$ phenol/trichloroethylene solution to dissolve the PET completely. Then, the dissolved polymer containing CNTs was precipitated by immersion in ethanol for $24 \mathrm{~h}$ in order to isolate the CNT/polymer composite and eliminate the phenol. Finally, the solid polymer was separated by filtration. The material obtained was cut into sections of approximately $15 \times 15 \mathrm{~mm}^{2}$ and placed in $700 \mathrm{~mL}$ of ethanol for $24 \mathrm{~h}$ to dissolve any residual phenol in the polymer, and then the process was repeated by using distilled water. The washed material was placed in a convection oven at $100^{\circ} \mathrm{C}$ for $48 \mathrm{~h}$. After $48 \mathrm{~h}$ of drying, the obtained films were pulverized and dried at $150^{\circ} \mathrm{C}$ for $4 \mathrm{~h}$. The second major step of the SSE method consisted in processing the obtained MWCNT/PET powder by direct extrusion (once), following the same conditions as for the DE method.

2.3. Characterization. Tensile properties of MWCNT/PET composites were investigated using a Shimadzu AGI-100 universal testing machine with a load cell of $0.5 \mathrm{kN}$ capacity. The tensile specimens used were circular rods of $150 \mathrm{~mm}$ length and $\sim 2 \mathrm{~mm}$ diameter directly obtained from the extruder. The gauge length (distance between the jaws) was $100 \mathrm{~mm}$, which was set by wrapping masking tape at the specimen edges to define end tabs. The measurements were carried out at a cross-head speed of $5 \mathrm{~mm} / \mathrm{min}$ and ten replicates were tested for each processing method.

The DC electrical conductivity of the MWCNT/PET composites was measured using a 6517B Keithley electrometer at room temperature. Due to their insulating character, samples with MWCNT concentrations less than $1 \mathrm{wt} \%$ required the use of a special guarding device (Keithley 8009 fixture). Since this device requires film geometry, the samples used for these MWCNT concentrations were films of $50 \mathrm{~mm}$ diameter and $\sim 1 \mathrm{~mm}$ thickness obtained by hot compressing the composite rods. The Raman spectra of the composites 
were obtained using a Raman spectrophotometer Kaiser 1.8i using a spectral resolution of $0.3 \mathrm{~cm}^{-1}$ and $\sim 17$ minutes of analysis per sample. The excitation source was a helium-neon laser with wavelength of $632.8 \mathrm{~nm}$ and $35 \mathrm{~mW}$ power.

Thermal properties of the composite materials processed by DE, MCE, and SSE were investigated by a Perkin Elmer Diamond DSC equipment. All samples were dried prior to the measurements and analyses were conducted in a nitrogen atmosphere using standard aluminum pans. The samples were exposed to heating temperature scans at a rate of $10^{\circ} \mathrm{C} / \mathrm{min}$ from 50 to $300^{\circ} \mathrm{C}$. The amount of crystallinity $\left(X_{c}\right)$ was obtained from the enthalpy measurements as

$$
X_{c}=\frac{\Delta H_{f}}{\left(1-M_{\mathrm{MWCNT}}\right) \Delta H_{f}^{0}} \text {, }
$$

where $M_{\text {MWCNT }}$ is the CNT fraction in the composite, $\Delta H_{f}$ is the measured fusion enthalpy (which is obtained from the area under the curve of the melting peak), and $\Delta H_{f}^{0}=$ $140 \mathrm{~J} / \mathrm{g}$ is the heat of fusion for a hypothetical 100\% crystalline PET [19]. To erase thermal history effects, the samples were subjected to two heating scans and the second scan is reported.

The fracture surfaces of the tested composites were examined by using a JEOL 6360LV SEM, after coating the surface with a thin layer of gold.

\section{Results and Discussion}

3.1. Mechanical Properties. The tensile stress-strain curves of neat PET and $0.1 \mathrm{wt} \%$ composites processed by DE, MCE, and SSE are presented in Figure 2. Material toughness was measured as the area under the stress-strain curve. Table 1 presents a summary of the measured mechanical properties, where average and standard deviation values are reported. The failure strain of PET processed by identical conditions than DE is large $(>25 \%)$ and such a large failure strain is greatly reduced by the inclusion of a stiff nanostructure such as the MWCNTs. The reduction, however, is less pronounced for materials processed by SSE or MCE than for DE. The reason for this is that MWCNTs in the PET matrix behave as physical crosslinking points, restricting the movement of polymer chains. Composites processed by DE and SSE showed similar tensile strength than the neat PET. However, composites processed by MCE showed an increase in tensile strength. This is possibly due to the more uniform dispersion of MWCNTs in the PET matrix, given the twostep process used for MWCNT dispersion in MCE. This hypothesis will be further examined in connection with the Raman spectroscopy and SEM analysis. As a consequence of the increased strength, the toughness of the composites processed by MCE is higher than that processed by DE. Among the composites, however, the toughest material is that processed by SSE, given its larger deformation capability (failure strain). The incorporation of MWCNTs in the PET matrix yields a substantial decrease in the elastic modulus of the composites processed by SSE, a negligible change for MCE, and a slight tendency to increase for DE, with

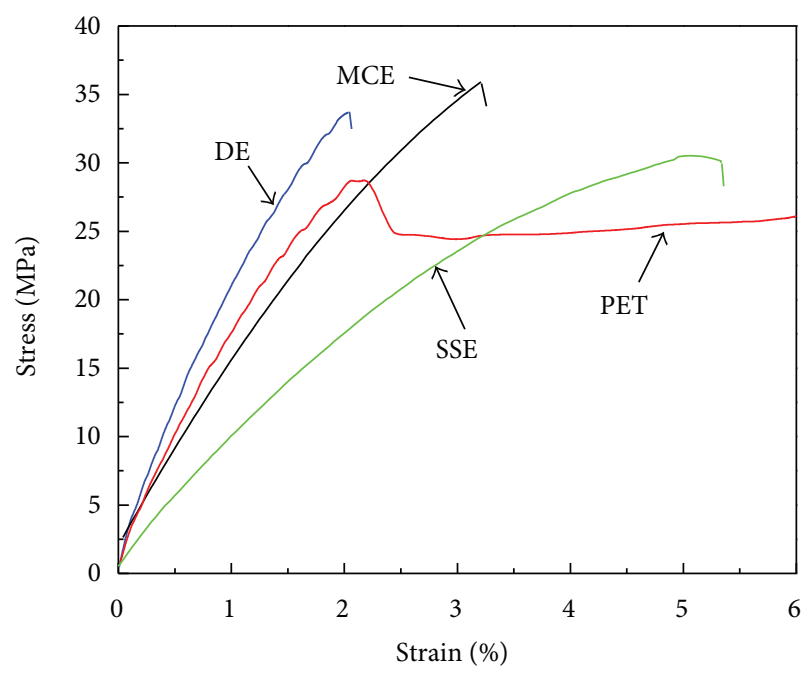

FIGURE 2: Typical stress-strain curves for PET and MWCNT/PET composites.

TABLE 1: Tensile mechanical properties of neat PET and MWCNT/PET composites containing $0.1 \mathrm{wt} \%$.

\begin{tabular}{lcccc}
\hline Material & $\begin{array}{c}\text { Tensile } \\
\text { strength } \\
(\mathrm{MPa})\end{array}$ & $\begin{array}{c}\text { Elastic } \\
\text { modulus } \\
(\mathrm{GPa})\end{array}$ & $\begin{array}{c}\text { Failure strain } \\
(\%)\end{array}$ & $\begin{array}{c}\text { Toughness } \\
\left(\mathrm{MJ} / \mathrm{m}^{3}\right)\end{array}$ \\
\hline PET & $30.5 \pm 3.26$ & $1.33 \pm 0.07$ & $25.0 \pm 6.30$ & $5.98 \pm 1.27$ \\
DE & $29.7 \pm 4.11$ & $1.50 \pm 0.24$ & $2.41 \pm 0.30$ & $0.47 \pm 0.04$ \\
MCE & $36.0 \pm 2.35$ & $1.22 \pm 0.13$ & $3.28 \pm 0.27$ & $0.63 \pm 0.19$ \\
SSE & $29.6 \pm 1.27$ & $0.73 \pm 0.02$ & $4.93 \pm 0.24$ & $0.93 \pm 0.12$ \\
\hline
\end{tabular}

respect to neat PET. It is noticed that the elastic modulus of composites processed by SSE decreases by about $45 \%$ with respect to neat $\mathrm{PET}$, with the concomitant increase in failure strain. This finding suggests that the solvent used in SSE may be causing matrix plasticization, which will be further investigated. MWCNT/PET composite with higher MWCNT concentrations ( 0.5 and $1.0 \mathrm{wt} \%)$ produced materials with increased elastic modulus and reduced failure strain (not discussed herein).

3.2. Electrical Conductivity. Figure 3 shows the DC electrical conductivity of composite materials processed by $\mathrm{DE}$ and MCE at MWCNT weight concentrations between 0.1 and $9 \mathrm{wt} \%$. Composites processed by SSE were not fabricated for such a large amount of MWCNT concentrations since this processing method is cumbersome and no significant changes in conductivity with respect to the other two methods were observed for composites with MWCNT concentrations less than $1 \mathrm{wt} \%$. Regardless of the processing method, for MWCNT weight concentrations below $1 \%$ no significant changes in conductivity were detected with respect to the neat polymer. Around $1 \mathrm{wt} \%$, the conductivity markedly increased indicating percolation. At $1 \mathrm{wt} \%$ the conductivity 


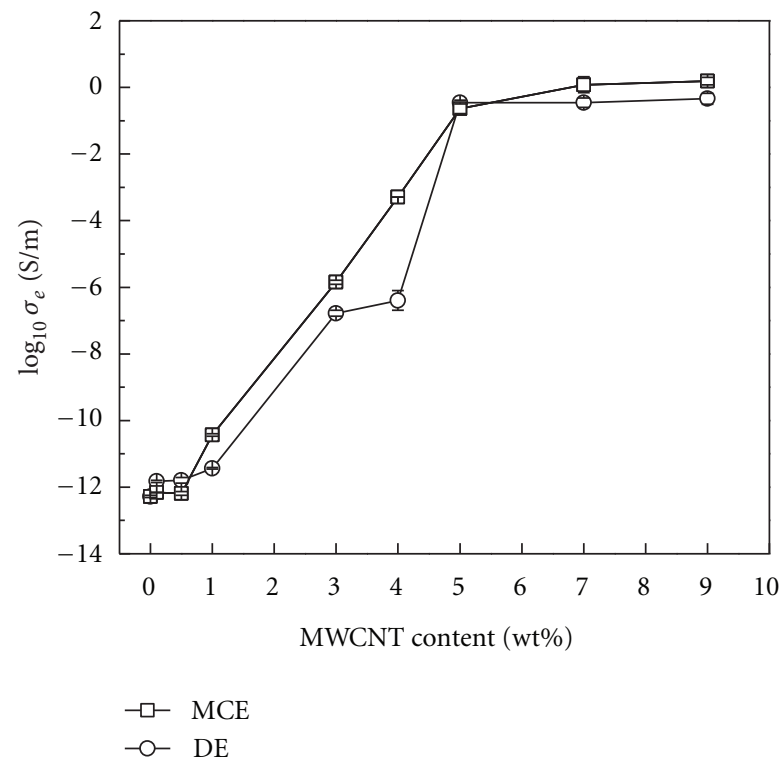

FIGURE 3: Electrical conductivity of MWCNT/PET composites processed by MCE and DE.

of the composites processed by MCE is almost one order of magnitude higher than that of composites processed by DE, suggesting that for MCE the onset of electrical percolation may correspond to this value, while slightly higher MWCNT concentrations may be needed to form a percolated network when using the DE processing method. For $3 \mathrm{wt} \%$ composites an increase in conductivity of 3-6 orders of magnitude is observed, indicating that a fully percolated network has already formed. For all composites at or above $1 \mathrm{wt} \%$ the conductivity of MCE is higher than that of DE. At $9 \mathrm{wt} \%$, the composites processed by MCE have a mean electrical conductivity of $1.54 \mathrm{Sm}^{-1}$, while the conductivity of the composites processed by $\mathrm{DE}$ is $0.45 \mathrm{Sm}^{-1}$. The difference between the conductivities of the composites processed by MCE and DE can be attributed to a better dispersion of the MWCNTs for MCE. This may be caused by unraveling the agglomerated MWCNTs by the shear forces exerted by the first processing step of MCE, namely, melt compounding.

3.3. Raman Spectroscopy. Raman spectroscopy was used as a tool to investigate the state of dispersion of MWCNTs in the composite matrix. Figure 4 shows the Raman spectra of the examined MWCNTs, PET, and $0.1 \mathrm{wt} \%$ composites processed by DE, MCE, and SSE. The MWCNTs display two characteristic bands at $\sim 1331 \mathrm{~cm}^{-1}$ and $\sim 1615 \mathrm{~cm}^{-1}$. The first band at $\sim 1331 \mathrm{~cm}^{-1}$ is assigned to the " $\mathrm{D}$ " band, which is derived from disordered graphite structures, while the second band at $1615 \mathrm{~cm}^{-1}$ is assigned to the " $\mathrm{G}$ " band, caused by the vibration of $\mathrm{C}-\mathrm{C}$ bonds in the graphitic structure
$[5,20]$. The spectrum of PET shows three intense bands at $1287 \mathrm{~cm}^{-1}$, corresponding to the vibration of $\mathrm{C}-\mathrm{O}$ bonds, $1618 \mathrm{~cm}^{-1}$, corresponding to the vibration of the aromatic ring in the main PET chain, and $1728 \mathrm{~cm}^{-1}$ corresponding to the vibration of $\mathrm{C}=\mathrm{O}$ groups [21]. Given the relatively low concentration of MWCNTs employed, the Raman spectra of all MWCNT/PET composites are similar to that of neat PET. The bands at 1728 and $1618 \mathrm{~cm}^{-1}$ are practically identical for neat PET or any of the composites, but a subtle difference can be noted for the band at $1287 \mathrm{~cm}^{-1}$. The wide $\mathrm{C}-\mathrm{O}$ band of PET at $1287 \mathrm{~cm}^{-1}$ overlaps with the intense D band of the nanotubes at $1331 \mathrm{~cm}^{-1}$. Therefore, a wide $\mathrm{C}-\mathrm{O}$ band (PET) with a shoulder (MWCNT D band) is observed for the composites processed by SSE and MCE, but such a shoulder is not observed for DE.

The reason for the presence of such a shoulder for SSE and MCE can be attributed to better dispersion of the MWCNTs within the polymer matrix, and such an interpretation can be better explained with the aid of the diagram in Figure 5. This figure shows schematically what is hypothesized as the relationship between the state of dispersion of the MWCNTs within the polymer matrix and its Raman spectra (Figure 4). For composites with reasonably homogenous CNT dispersion (such as those processed by MCE and SSE, Figure 5(a)), there is a high probability that the Raman analysis captures the signal of the MWCNTs within the composite, since several MWCNTs should be present in the analysis zone. However, for composites with larger MWCNT agglomerates, there is a higher chance that no nanotubes are present in 


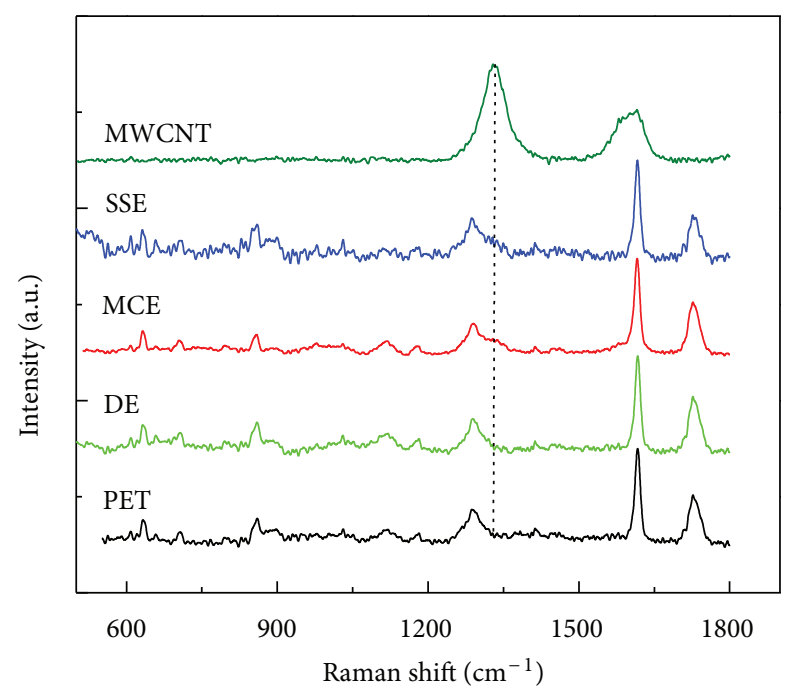

FIGURE 4: Raman spectra of MWCNTs, PET, and MWCNT/PET composites.

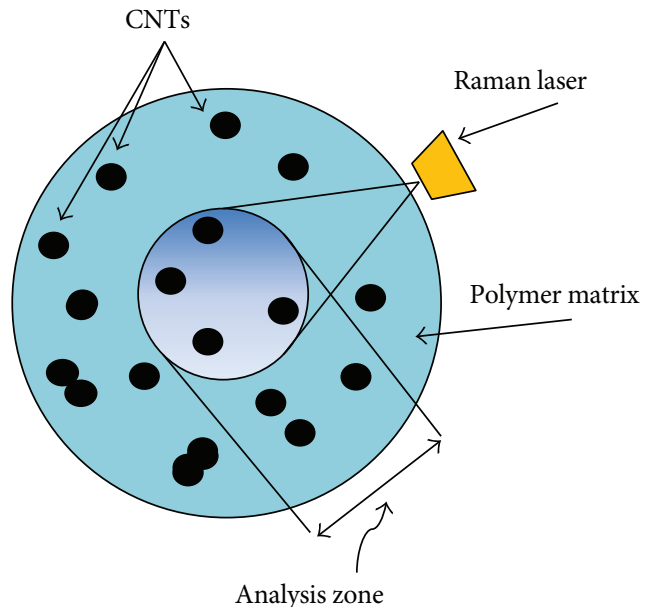

(a)

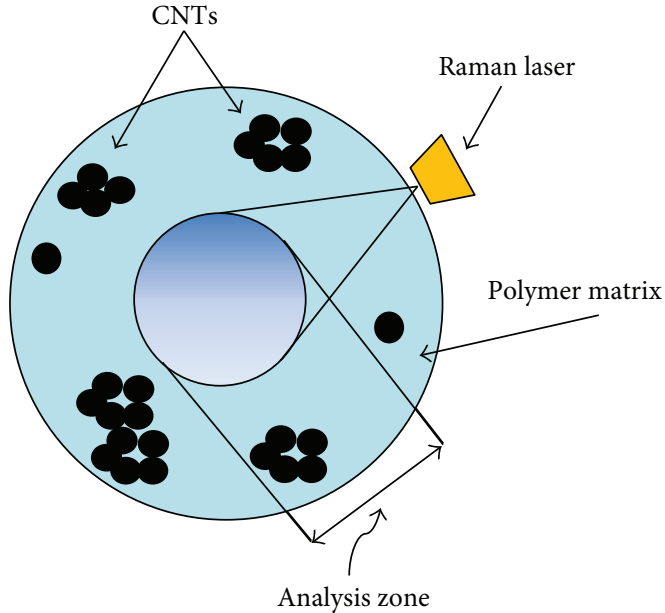

(b)

FIGURE 5: Schematic representation of MWCNT dispersion in the polymer matrix for interpretation of the Raman spectra. (a) Composites fabricated by MCE and SSE, (b) composites fabricated by DE.

the analysis zone (Figure 5(b)), so the Raman signal of those MWCNT composites is indistinguishable from the Raman signal of the polymer.

3.4. Melting and Crystallization Behavior. The melting and crystallization behavior of MWCNT/PET composites was analyzed by nonisothermal DSC. Figure 6 shows the DSC thermograms of PET and all composites investigated at $0.1 \mathrm{wt} \%$, and the DSC results are summarized in Table 2.

Figure 6 shows that, for all MWCNT/PET composites, the peak position corresponding to the melting temperature $\left(T_{m}\right)$ is similar for the three processing methods investigated. However, there is a small shoulder at the base of the melting peak (on the left side) for composites, which is not present in the thermogram of PET. This shoulder is attributed to the addition of MWCNTs to PET, and its presence suggests the formation of PET crystals that melt at lower temperatures by the presence of CNTs $[22,23]$, indicating that the CNTs act as nucleation sites for PET crystals.

Table 2 shows the results of the crystallization temperature $\left(T_{c}\right)$, glass-transition temperature $\left(T_{g}\right)$, and melting temperature $\left(T_{m}\right)$ of the MWCNT/PET composites. $T_{m}$ is similar in all cases, indicating that the PET crystals in the composites are melted at a similar average temperature. As discussed previously, the small shoulder at temperatures slightly lower than $T_{m}$, however, suggests that some crystals are wrapped around the MWCNTs and melt at lower temperatures. The incorporation of MWCNTs in the PET matrix has a small effect on the crystallization temperature $\left(T_{c}\right)$ and such an effect is more notorious for the composites obtained 


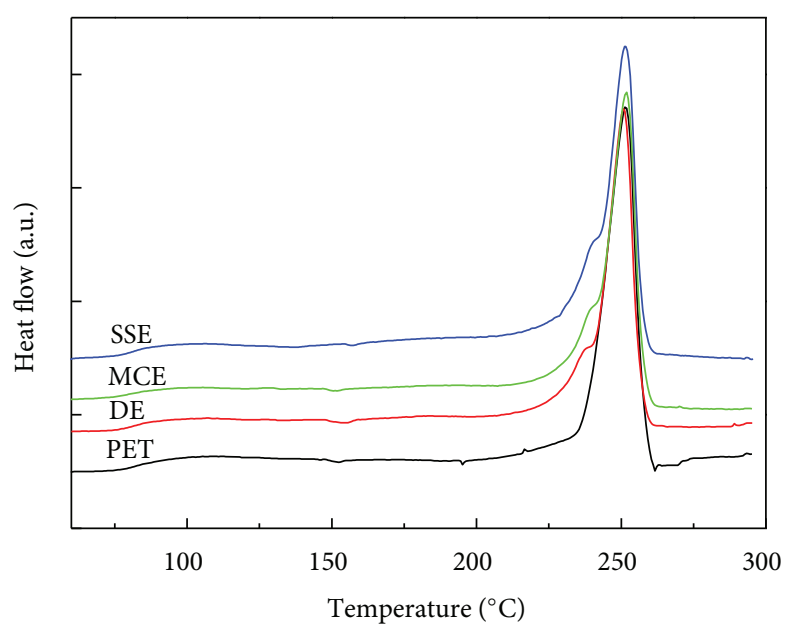

FIGURE 6: DSC thermograms of neat PET and $0.1 \mathrm{wt} \%$ MWCNT/PET composites.

TABLE 2: DSC thermal characteristics of neat PET and its composites containing $0.1 \mathrm{wt} \%$ MWCNTs.

\begin{tabular}{lccccc}
\hline Material & $\begin{array}{c}T_{g} \\
\left({ }^{\circ} \mathrm{C}\right)\end{array}$ & $\begin{array}{c}T_{m} \\
\left({ }^{\circ} \mathrm{C}\right)\end{array}$ & $\begin{array}{c}T_{c} \\
\left({ }^{\circ} \mathrm{C}\right)\end{array}$ & $\begin{array}{c}\Delta H_{f} \\
(\mathrm{~J} / \mathrm{g})\end{array}$ & $\begin{array}{c}X_{c} \\
(\%)\end{array}$ \\
\hline PET & 85.5 & 251.7 & 152.0 & 41.5 & 58.1 \\
DE & 83.8 & 251.1 & 154.4 & 38.0 & 53.2 \\
MCE & 84.6 & 252.5 & 152.3 & 39.5 & 55.3 \\
SSE & 83.2 & 251.7 & 156.9 & 41.6 & 58.2 \\
\hline
\end{tabular}

by the SSE method. The slight increase in the crystallization temperature of the composites may also be attributed to the nucleating effects of the MWCNTs. The presence of MWCNTs decreases the mobility of the polymer chains in the composite material and hence reduces their ductility; see, for example, [24]. The glass transition temperature $\left(T_{g}\right)$ of the composites processed by the SSE method decreases with respect to the neat PET; this means that polymer chains become flexible at lower temperatures compared to PET, and composites processed by MCE and DE. This decrease in $T_{g}$ can be attributed to a plasticizing effect on the polymer chains due to the dissolution-solidification process, as well as possible trapped solvent, causing an increase in the free volume of the polymer. The plasticizing effect is in agreement with the results of the mechanical tests discussed in Section 3.1.

Previous works $[25,26]$ explain the mechanism of crystallization of a semicrystalline polymer such as PET using the description of two phases (amorphous and crystalline). However, more recent studies $[27,28]$ pinpoint that this model does not adequately describe the structure of many of such polymers. They claim that a model with three phases, which comprises a crystalline phase $(\mathrm{CP})$, a mobile amorphous fraction (MAF), and rigid amorphous fraction (RAF), is more adequate to describe the melting and crystallization behavior of PET. The RAF is considered an "intermediate stage" between the amorphous and crystalline phases [28].
The RAF has no contribution to the heat of fusion of crystals, as well as to the glass transition temperature of the MAF, and therefore only contributes to the physical properties of the polymer. PET is a typical semicrystalline polymer with a relatively high amount of RAF. Previous works [25] report RAF for PET in the range of 11 to $44 \%$. The presence of MWCNTs suggests a change in the proportion of these phases, since the carbon nanotubes act as nucleating agents [29].

3.5. Examination of Fracture Surfaces. Figure 7 shows SEM images of $0.1 \mathrm{wt} \%$ MWCNT/PET composites processed by DE (first row), MCE (second row), and SSE (third row). As seen from the low magnification images (first column), the method employed for manufacturing the composites affects their fracture surface morphology. The fracture surface of composites processed by DE is flat and smooth, with only small ridges, characteristic of a brittle fracture. A rougher surface with more ridges distributed over the fracture surface is observed for composites processed by MCE, indicating a more ductile fracture. Since the only difference between DE and MCE is the processing method, these differences are attributed to a more homogeneous dispersion of the MWCNTs within the PET matrix. For the material processed by SSE, large ductility and plasticity are evident by the significant necking of the cross-section, once again suggesting matrix plasticization. These observations are in agreement with the results obtained in the mechanical characterization. The tips of the MWCNTs covered by a thin layer of PET are observed as bright dots in the high magnification images (second column of Figure 7). A more uniform distribution of MWCNTs is observed for the composites processed by MCE than for DE, which is in agreement with the results of the Raman spectroscopy analysis. The MWCNTs are not visible for composites processed by SSE. Instead, a rough and irregular polymer surface confirms that the residual solvent used in this method yields PET plasticization, in agreement with the previous observations. 

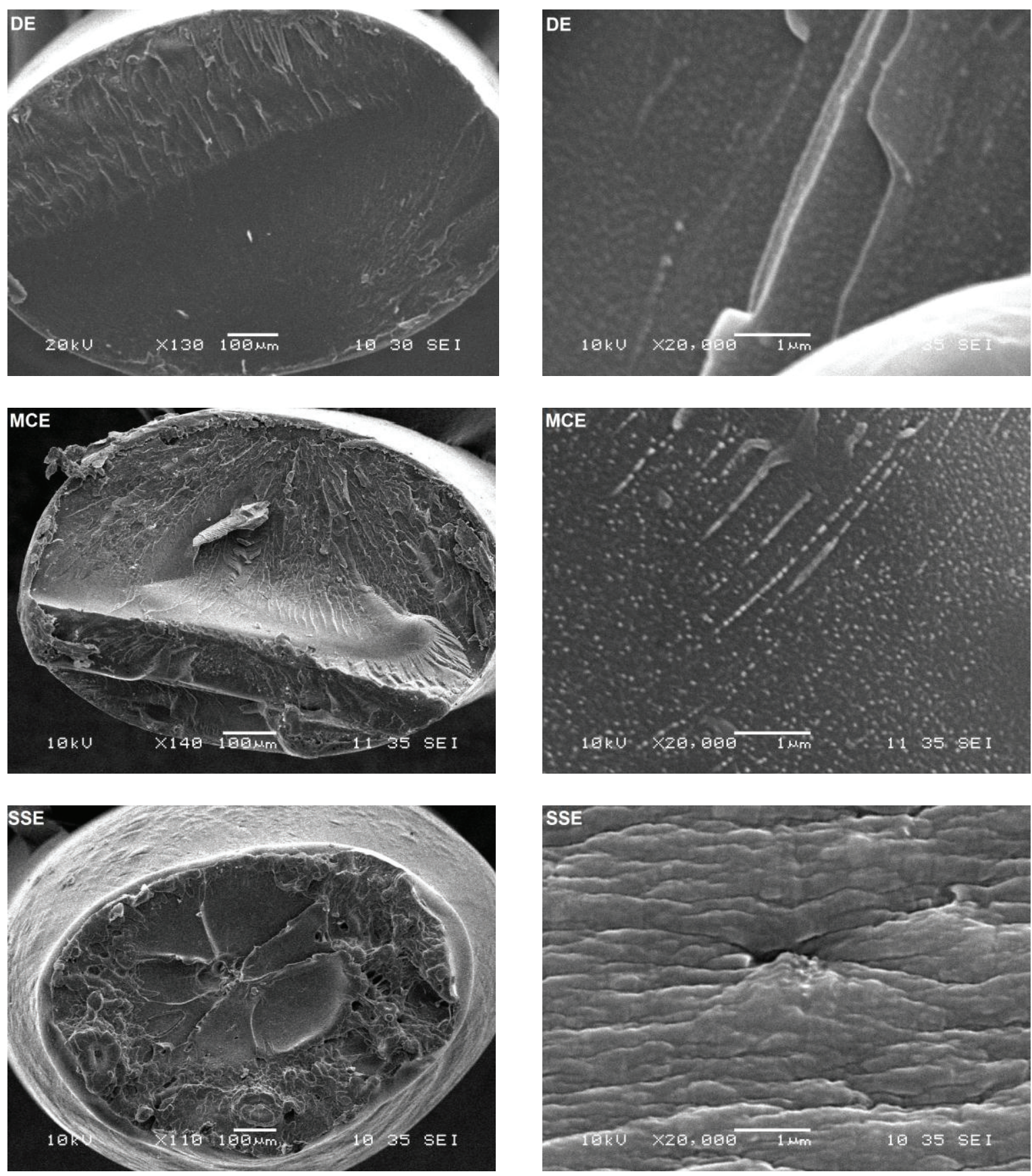

FiguRE 7: SEM images of fracture surfaces of $0.1 \mathrm{wt} \%$ MWCNT/PET composites. Left column corresponds to low magnification $(\sim \times 100)$, and right column to high magnification $(\times 20,000)$.

\section{Conclusions}

MWCNT/PET composites were prepared following three different processing methods which include extrusion as their final step: direct extrusion (DE), melt compounding followed by extrusion (MCE), and dispersion of the CNTs in a solvent by ultrasonication followed by extrusion (SSE). Inclusion of $0.1 \mathrm{wt} \%$ MWCNTs into the PET matrix made the polymer stiffer but reduced its elongation at break and consequent toughness, especially for the composites processed by $\mathrm{DE}$.
The tensile strength of the composites processed by MCE increased with respect to the neat PET. DSC indicates that the MWCNTs act as nucleating agents and also points out to matrix plasticization for the case of the SSE processing method. Electron microscopy reveals a brittle fracture for composites processed by DE and a less brittle fracture for composites processed by MCE and SSE (with respect to DE). Electrical percolation occurred around $1 \mathrm{wt} \%$ for composites processed by DE and MCE and electrical conductivity after percolation was higher for composites manufactured 
by the MCE method. At $9 \mathrm{wt} \%$, the composites processed by MCE have a mean electrical conductivity of $1.54 \mathrm{Sm}^{-1}$, while the conductivity of the composites processed by $\mathrm{DE}$ is $0.45 \mathrm{Sm}^{-1}$. Raman spectroscopy and SEM analyses indicate that the cause of these improvements for MCE is a more homogeneous dispersion and distribution of the MWCNTs into the PET matrix. Therefore, melt compounding of the MWCNT/PET polymer before extrusion is recommended to improve the mechanical and electrical properties of the composites. Polymer processing assisted by solvents may yield good dispersion of the MWCNTs, but it is not recommended since it is prone to yield plasticization of the polymer matrix and is cumbersome to scale-up.

\section{Conflict of Interests}

The authors do not have any conflict of interests regarding the companies which provided the raw materials and fabricated the equipments used in this research and are not related to any of those companies.

\section{Acknowledgments}

This work was part of an international project between Mexico and Chile (CIAM) sponsored by CONACYT-Mexico and CONICYT-Chile (Project FONDECYT no. 1090260). Mr. O. Rodríguez-Uicab acknowledges the scholarship received from CONACYT. The authors also wish to thank Rossana Vargas and Ronny Schönfelder for the SEM and Raman analyses, as well as Dr. Juan Cauich for his technical advice.

\section{References}

[1] D. W. Van Krevelen, Properties of Polymers, Elsevier, Amsterdam, The Netherlands, 1997.

[2] J. M. G. Cowie, Polymers: Chemistry and Physics of Modern Materials, Taylor and Francis Group, Boca Raton, Fla, USA, 2008.

[3] E. Logakis, P. Pissis, D. Pospiech et al., "Low electrical percolation threshold in poly(ethylene terephthalate)/multi-walled carbon nanotube nanocomposites," European Polymer Journal, vol. 46, no. 5, pp. 928-936, 2010.

[4] G. Sun, G. Chen, Z. Liu, and M. Chen, "Preparation, crystallization, electrical conductivity and thermal stability of syndiotactic polystyrene/carbon nanotube composites," Carbon, vol. 48, no. 5, pp. 1434-1440, 2010.

[5] S. H. Jin, Y. B. Park, and K. H. Yoon, "Rheological and mechanical properties of surface modified multi-walled carbon nanotube-filled PET composite," Composites Science and Technology, vol. 67, no. 15-16, pp. 3434-3441, 2007.

[6] O. Breuer and U. Sundararaj, "Big returns from small fibers: a review of polymer/carbon nanotube composites," Polymer Composites, vol. 25, no. 6, pp. 630-645, 2004.

[7] Z. Zhu, R. Wang, Z. Dong, X. Huang, and D. Zhang, "Morphology, crystallization, and mechanical properties of poly(ethylene terephthalate)/multiwalled carbon nanotubes composites," Journal of Applied Polymer Science, vol. 120, no. 6, pp. 3460-3468, 2011.
[8] J. Y. Kim, H. S. Park, and S. H. Kim, "Multiwall-carbonnanotube-reinforced poly(ethylene terephthalate) nanocomposites by melt compounding," Journal of Applied Polymer Science, vol. 103, no. 3, pp. 1450-1457, 2007.

[9] A. K. Anand, U. S. Agarwal, A. Nisal, and R. Joseph, "PETSWNT nanocomposites through ultrasound assisted dissolution-evaporation," European Polymer Journal, vol. 43, no. 6, pp. 2279-2285, 2007.

[10] M. Mukherjee, T. Das, R. Rajasekar, S. Bose, S. Kumar, and C. K. Das, "Improvement of the properties of PC/LCP blends in the presence of carbon nanotubes," Composites A, vol. 40, no. 8, pp. 1291-1298, 2009.

[11] P. C. Ma, N. A. Siddiqui, G. Marom, and J. K. Kim, "Dispersion and functionalization of carbon nanotubes for polymer-based nanocomposites: a review," Composites $A$, vol. 41, no. 10, pp. 1345-1367, 2010.

[12] N. G. Sahoo, S. Rana, J. W. Cho, L. Li, and S. H. Chan, "Polymer nanocomposites based on functionalized carbon nanotubes," Progress in Polymer Science, vol. 35, no. 7, pp. 837-867, 2010.

[13] Z. Jia, Z. Wang, C. Xu et al., "Study on poly(methyl methacrylate)/carbon nanotube composites," Materials Science and Engineering A, vol. 271, no. 1-2, pp. 395-400, 1999.

[14] D. Bikiaris, A. Vassiliou, K. Chrissafis, K. M. Paraskevopoulos, A. Jannakoudakis, and A. Docoslis, "Effect of acid treated multiwalled carbon nanotubes on the mechanical, permeability, thermal properties and thermo-oxidative stability of isotactic polypropylene," Polymer Degradation and Stability, vol. 93, no. 5, pp. 952-967, 2008.

[15] M. O. Lisunova, Y. P. Mamunya, N. I. Lebovka, and A. V. Melezhyk, "Percolation behaviour of ultrahigh molecular weight polyethylene/multi-walled carbon nanotubes composites," European Polymer Journal, vol. 43, no. 3, pp. 949-958, 2007.

[16] G. Santoro, M. A. Gómez, C. Marco, and G. Ellis, "A solventfree dispersion method for the preparation of PET/MWCNT composites," Macromolecular Materials and Engineering, vol. 295, no. 7, pp. 652-659, 2010.

[17] K. A. Anand, U. S. Agarwal, and R. Joseph, "Carbon nanotubesreinforced PET nanocomposite by melt-compounding," Journal of Applied Polymer Science, vol. 104, no. 5, pp. 3090-3095, 2007.

[18] F. Avilés, A. Ponce, J. V. Cauich-Rodríguez, and G. T. Martínez, "TEM examination of MWCNTs Oxidized by mild experimental conditions," Fullerenes, Nanotubes, Carbon Nanostructures, vol. 20, pp. 49-55, 2012.

[19] H. Chen, Z. Liu, and P. Cebe, "Chain confinement in electrospun nanofibers of PET with carbon nanotubes," Polymer, vol. 50, no. 3, pp. 872-880, 2009.

[20] T. McNally, P. Pötschke, P. Halley et al., "Polyethylene multiwalled carbon nanotube composites," Polymer, vol. 46, no. 19, pp. 8222-8232, 2005.

[21] K. Awasthi, V. Kulshrestha, D. K. Avasthi, and Y. K. Vijay, "Optical, chemical and structural modification of oxygen irradiated PET," Radiation Measurements, vol. 45, no. 7, pp. 850-855, 2010.

[22] Y. Kong and J. N. Hay, "Multiple melting behaviour of poly(ethylene terephthalate)," Polymer, vol. 44, no. 3, pp. 623-633, 2002.

[23] I. Okazaki and B. Wunderlich, "Reversible melting in polymer crystals detected by temperature-modulated differential scanning calorimetry," Macromolecules, vol. 30, no. 6, pp. 1758-1764, 1997. 
[24] G. Antoniadis, K. M. Paraskevopoulos, D. Bikiaris, and K. Chrissafis, "Kinetics study of cold-crystallization of poly(ethylene terephthalate) nanocomposites with multi-walled carbon nanotubes," Thermochimica Acta, vol. 493, no. 1-2, pp. 68-75, 2009.

[25] T. McNally, P. Pötschke, P. Halley et al., "Polyethylene multiwalled carbon nanotube composites," Polymer, vol. 46, no. 19, pp. 8222-8232, 2005.

[26] R. Androsch and B. Wunderlich, "The link between rigid amorphous fraction and crystal perfection in cold-crystallized poly(ethylene terephthalate)," Polymer, vol. 46, no. 26, pp. 12556-12566, 2005.

[27] M. Arnoult, E. Dargent, and J. F. Mano, "Mobile amorphous phase fragility in semi-crystalline polymers: comparison of PET and PLLA," Polymer, vol. 48, no. 4, pp. 1012-1019, 2007.

[28] S. Tzavalas and V. G. Gregoriou, "Uniaxially stretched PET/PET-MWNT nanocomposites: effect of the MWNTs on the chain conformations of PET," Vibrational Spectroscopy, vol. 46, no. 2, pp. 135-140, 2008.

[29] Z. Li, G. Luo, F. Wei, and Y. Huang, "Microstructure of carbon nanotubes/PET conductive composites fibers and their properties," Composites Science and Technology, vol. 66, no. 7-8, pp. 1022-1029, 2006. 

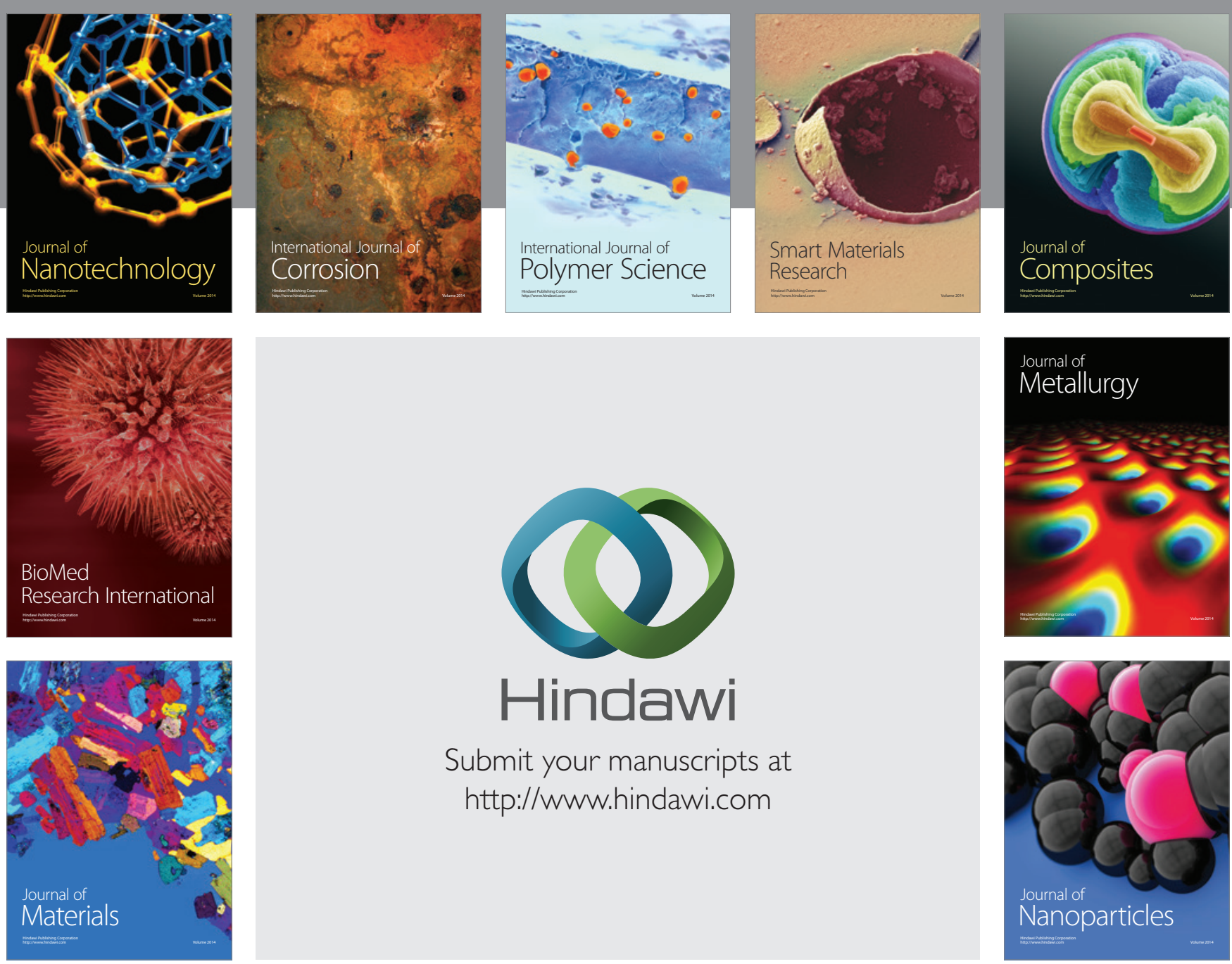

Submit your manuscripts at http://www.hindawi.com
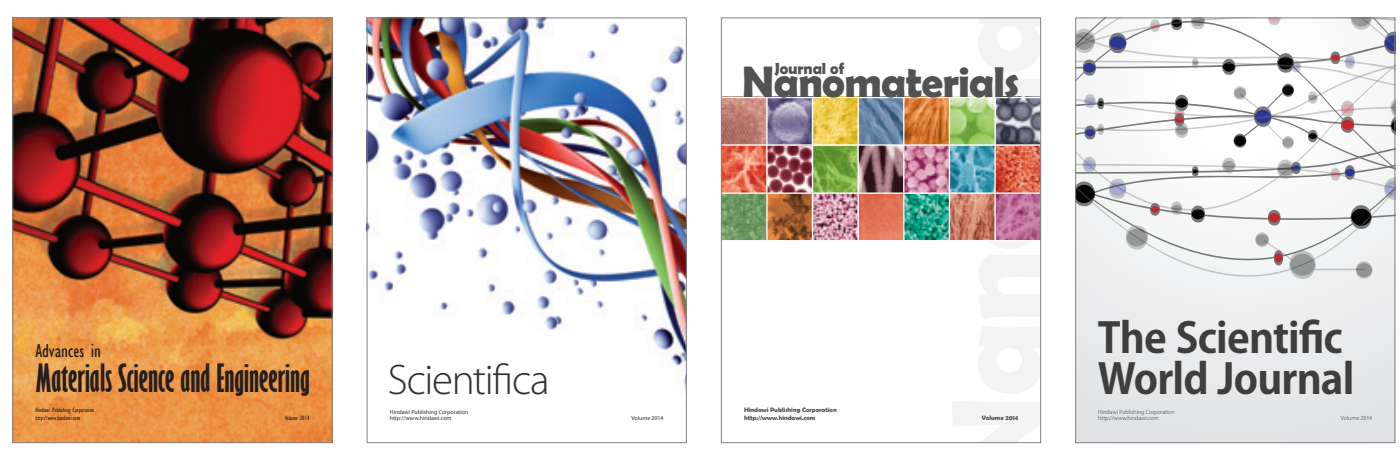

\section{The Scientific World Journal}
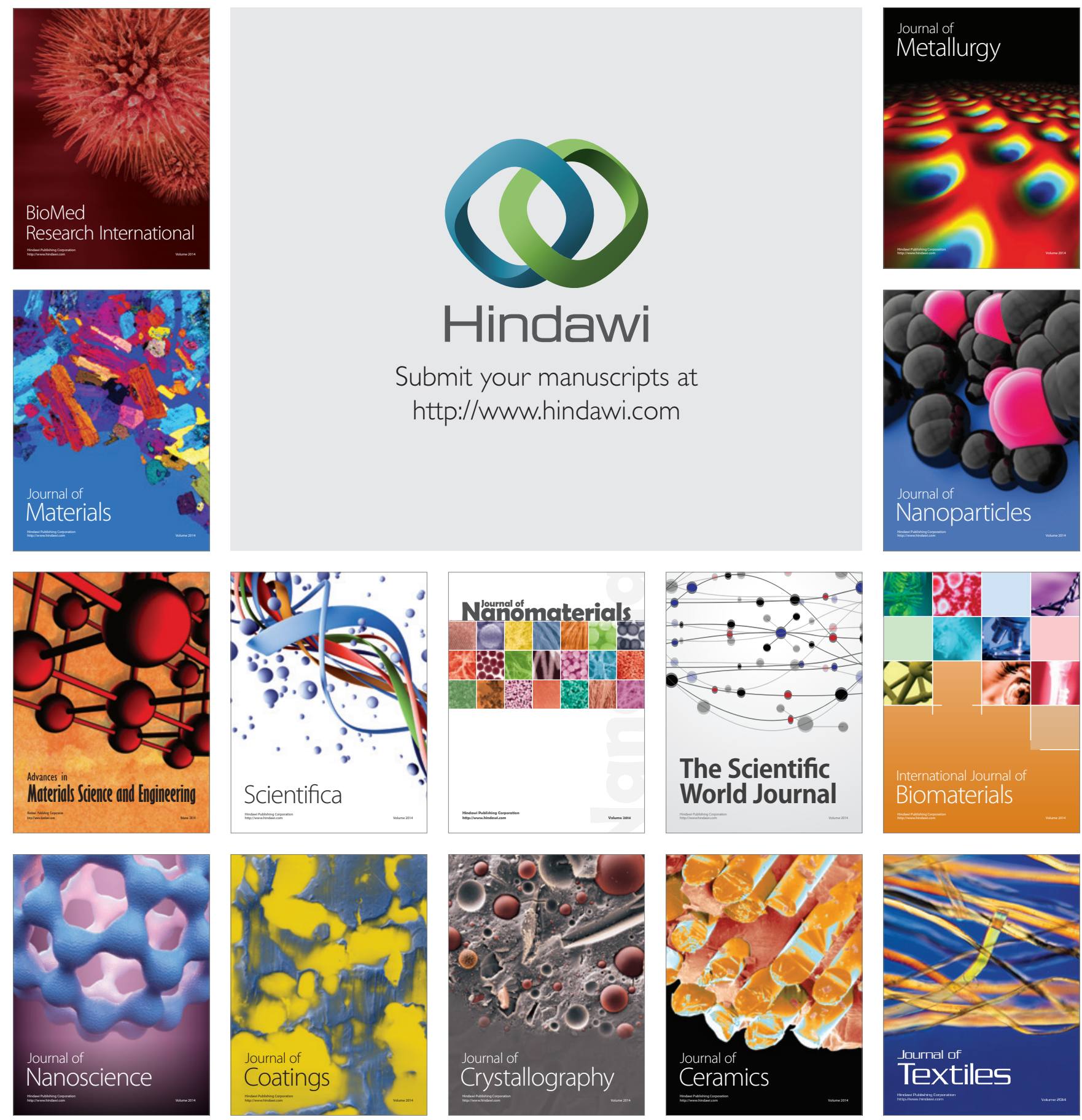